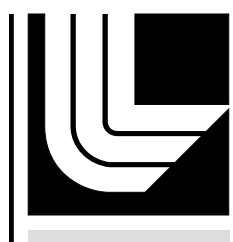

LAWRENCE LIVERM ORE NATION AL LABORATORY

X-ray diffraction studies of dynamically compressed diamond

J. Eggert

June 11, 2010 
This document was prepared as an account of work sponsored by an agency of the United States government. Neither the United States government nor Lawrence Livermore National Security, LLC, nor any of their employees makes any warranty, expressed or implied, or assumes any legal liability or responsibility for the accuracy, completeness, or usefulness of any information, apparatus, product, or process disclosed, or represents that its use would not infringe privately owned rights. Reference herein to any specific commercial product, process, or service by trade name, trademark, manufacturer, or otherwise does not necessarily constitute or imply its endorsement, recommendation, or favoring by the United States government or Lawrence Livermore National Security, LLC. The views and opinions of authors expressed herein do not necessarily state or reflect those of the United States government or Lawrence Livermore National Security, LLC, and shall not be used for advertising or product endorsement purposes.

This work performed under the auspices of the U.S. Department of Energy by Lawrence Livermore National Laboratory under Contract DE-AC52-07NA27344. 
National Ignition Facility (NIF)

Facility Time Proposal

\section{X-ray Diffraction Studies of Dynamically Compressed Diamond}

\section{Purpose:}

We propose a series of experiments to use X-ray diffraction (XRD) to study material properties using the NIF. XRD is the best way to determine the structure, lattice deformation, and texture of materials. Advances in synchrotron XRD facilities in the past two decades have revolutionized the study of materials at static high pressure in diamond anvil cells (DACs) up to about 3 Mbar. The National Ignition Facility (NIF) has the potential to do the same for dynamic materials studies at high-pressure. Members of our scientific team have pioneered XRD on many smaller laser facilities around the world. Our results suggest that diffraction on solids approaching $100 \mathrm{Mbar}$ may be possible on the NIF, providing access to new regime for matter at extreme conditions.

\section{Scientific Case:}

An understanding of the fundamental physics of the response of matter to rapid compression - be it shock or quasi-isentropic - remains an important, elusive challenge in the fields of laser-matter interactions and high energy-density physics. The capabilities of the NIF greatly expand capabilities in this burgeoning field, as the high energy and long shaped-pulse capability of this unique platform will allow the precise temporally-controlled compression of matter to many tens of Mbars, heralding a new era of high-pressure materials science, with the potential to uncover hitherto unknown highpressure crystalline phases and material properties.

The aim of this proposal is to reach an understanding, at the atomic level, of the pathways taken to, and the final forms of, these new states of matter. The diagnostic of choice is nanosecond X-ray diffraction. This proven technique, pioneered on more modest laser platforms (at consequently lower pressures) by the applicants, affords the potential for direct monitoring of lattice deformation before, during, and after polymorphic phase transitions. We are firmly of the view that dynamic ultra-highpressure materials science on NIF will be an extremely fruitful area of study. There are many materials that we wish to interrogate as the field matures, with varying, but equally compelling scientific rationales. This consortium proposes an initial concentration on polycrystalline diamond targets using an angle dispersive camera.

There are two persuasive reasons for this approach. First, the beauty of materials with the diamond cubic structure is that we can obtain single-crystal samples which are, for most purposes, 'perfect' - i.e. dislocation free. There is a strong line of reasoning that indicates these materials are better suited for theoretical modeling, both in terms of ab-initio calculations, and large (multi-million to billion atom) classical molecular dynamics calculations. Second, low-Z, strong and/or highly anisotropic materials, such as diamond and beryllium remain as candidates for ablator materials for ICF targets. Depending upon the target and drive-pulse designs, material phase, strength, texture, and lattice response may play a role in the subsequent overall target performance - particularly if failure modes for the crystals occur on spatial scale-lengths 
that can seed the Rayleigh-Taylor instability. Thus, for this class of materials, there is excellent synergy between curiosity-driven, high-pressure materials science and NIF's ultimate programmatic goals.

Diamond has many technologically important properties including its metastability, high compressive strength, high thermal conductivity, excellent transparency in the visible, and the highest bulk modulus of any material. Diamond is an ideal material for extreme-pressure dynamic experiments as demonstrated by the number of recent experimental publications by our team ${ }^{1-6}$, and others ${ }^{7-9}$. For many years a series of phase transitions have been predicted to occur in carbon at pressures of 5 to $30 \mathrm{Mbar}$. The first of these phases, BC8 has recently been determined to be the lowest-energy structure above $10 \mathrm{Mbar},{ }^{10}$ and has been experimentally claimed based on very subtle changes in the Hugoniot slope. ${ }^{7}$ Unfortunately, the most recent molecular-dynamics ${ }^{11}$ and meta-dynamics ${ }^{12}$ simulations suggest that the diamond-BC8 transition may be very slow, or that diamond may remain metastable throughout the BC8 stability regime. It has recently been proposed, using state-of-the-art metadynamics simulations, that by compressing diamond to the SC4 phase at $\sim 20$ Mbar, then releasing the pressure to $\sim 10$ Mbar, the BC8 phase may be produced. ${ }^{12}$ It was also found ${ }^{12}$ that the $\mathrm{BC} 8$ phase is likely metastable at ambient conditions and may be recoverable at ambient pressure, as has been done with silicon. ${ }^{13}$ This was shown by a calculation of the phonons of BC8 carbon at ambient pressure (unpublished results). Very recent theoretical results suggest that the bulk and shear modulus (and therefore possibly the hardness) of recovered BC8 carbon would be similar to, or greater than that of diamond.

The only way to confirm these proposals is to do a direct structural determination

and recovery of the extreme pressure phases of carbon: precisely our proposed experiments on the NIF

\section{Experimental Feasibility:}

The experiments will be carried out on the 'materials science' NIF platform. Polycrystalline materials are interrogated with a collimated quasi-monochromatic X-ray source. We use helium-alpha emission from laser-produced plasma produced by a metallic foil or foam ${ }^{14}$ target made of a mid-Z element. Simultaneously with the diffraction data we will monitor the target compression using VISAR to obtain not only information about the crystal's phase and strain history, but also the stress profiles. The requirement for NIF is a relatively long (several ns to tens of ns) drive, with precision temporal shaping, in order to ultimately compress the material isentropically to pressures far above the normal point of melting on the shock Hugoniot. Our final goal is to observe the phase and response of diamond at between 20 and 30 Mbar.

\section{Methodology:}

We rely on our recent successes on Omega and Omega EP on diffraction of iron and diamond to $5 \mathrm{Mbar}$ to design and perform the NIF experiments. The critical advance in these experiments is the use of diamond-sandwich targets. Much as a static diamond-anvil cell (DAC) compresses the sample between two strong diamond anvils, we confine a thin $(\sim 4-20 \mu \mathrm{m})$ sample between two stiff diamond windows (Fig. 1). The first diamond acts as an ablator, interacting with the laser and transmitting a ramped- 


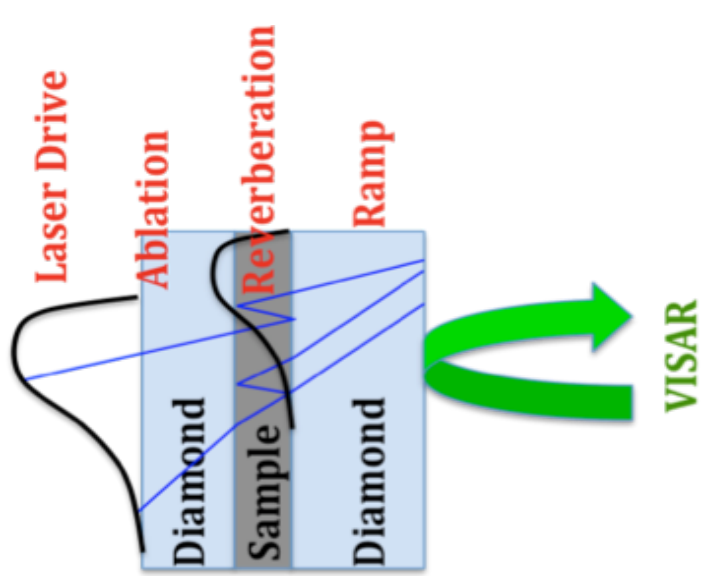

Fig. 1. Potential geometry and pulse-shape for initial optical measurements for ramp compressed diamond to $30 \mathrm{Mbar}$.

pressure drive to the package. The second diamond acts as a tamper, maintaining a high pressure in the thin sample until reverberations from the free surface return to release the sample pressure. At peak pressure we probe the conditions in the sample by carefully synchronizing a second laser pulse which produces our probe x-rays. Our primary pressure diagnostic is the free surface velocity history (of the rear surface) measured by VISAR. This and the particle velocity to pressure calibration derived from other experiments ${ }^{3}$ directly yields the pressure history in the sample. As long as the sample is sufficiently thin, it is a small perturbation to the hydrodynamics of the diamond confining the sample and the sample pressure closely follows that of the diamond windows. A slight perturbation of this geometry uses a LiF window in place of the second diamond. We have shown in experiments at Omega and Omega EP that LiF remains transparent to over 8 Mbar. ${ }^{15}$ This geometry would allow more direct examination of the sample pressure, synchronous optical measurements, and an estimate of the sample temperature. We have developed feasible NIF pulse shapes for diamond ramps to over $30 \mathrm{Mbar}$ and conceptual drives to $100 \mathrm{Mbar}$.

At Omega and Omega EP we have successfully fielded a powder x-ray diffraction image plate (PXRDIP) holder in five experiments (Fig. 2). We have measured diffraction on tin to $2 \mathrm{Mbar}$, on iron to $4.7 \mathrm{Mbar}$, and on diamond to over 5 Mbar. All three of these dynamic diffraction results significantly exceed the highestpressure static experiments and represent the highest-pressure diffraction ever measured. The PXRDIP was designed to minimize the need for precise relative alignment of the x-ray source and the sample. The critical alignment needs are that the lasers hit the x-ray source, and the sample independently. This is done routinely at Omega and the NIF.

The NIF will allow us to significantly increase both the maximum pressure and precision of these diffraction experiments. We propose three different XRD diagnostics for NIF shown in Fig. 3.. A) An image plate (IP) box very similar to the PXRDIP fielded at Omega. This geometry uses a halfraum drive geometry and a VISAR mirror mounted behind the back IP. All the unconverted light is incident on the horizontal front IP shield;
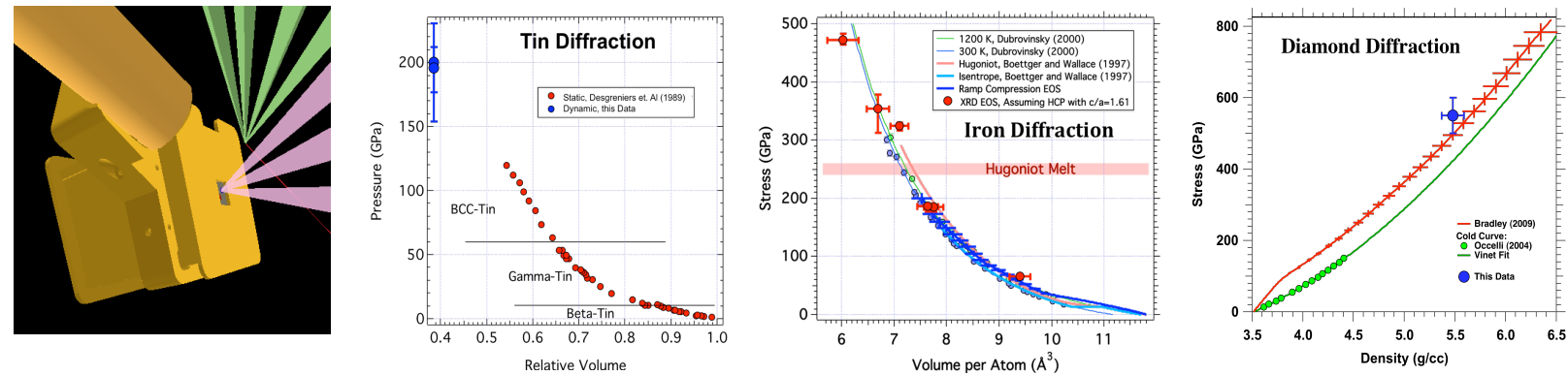

Fig. 2. X-ray diffraction configuration fielded at Omega. Unpublished diffraction results on solid tin, iron and diamond from Omega experiments. 
in order for the x-ray source to avoid the unconverted light it must be placed directly in line with the sample. B) A pyramid-shaped IP box modeled after the broad-band x-ray diffraction (BBXRD) diagnostic fielded on Omega by members of this team. This geometry allows a NIF hohlraum drive with the unconverted light incident on the slanted sides of the IP box. This configuration allows flexibility in the placement of the x-ray source while still avoiding the unconverted light. C) Finally, a Soller slit can shield the IP from unwanted x-radiation at the same time that the entire diagnostic is kept out of the unconverted light. This concept has not yet been tested on a laser platform, but has been shown to be very effective on high-pressure synchrotron beam lines. ${ }^{16}$ While the Soller slits eliminate problems associated with unconverted light, an effective alignment procedure needs to be developed. All three proposed diagnostics involve relatively large targets or diagnostics placed close to target-chamber center, and will require careful evaluation to be fielded at the NIF. We have begun evaluating these concepts with key members of the TALIS committee and are confident that by integrating NIF facility teams, a suitable XRD diagnostic package can be designed and fielded. There is a great programmatic needs for an XRD capacity on the NIF, and the substantial overlap between this team and the programmatic teams will ensure that an optimal diagnostic is fielded that is flexible enough to allow all desired XRD-based experiments to use a single diagnostic.

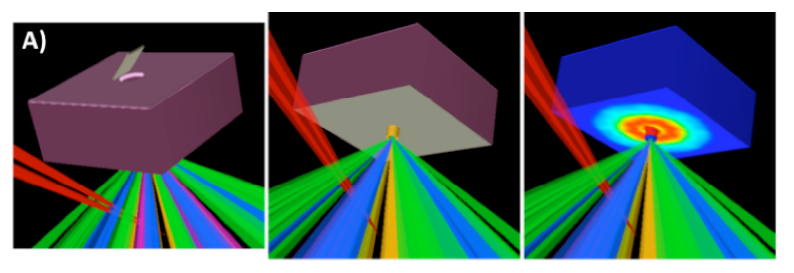

Fig. 3. Three possible configurations for an $\mathrm{x}-$ ray diffraction diagnostic on the NIF. A) IP box with hemispherical symmetry. Requires a $90^{\circ}$ VISAR mirror, on-axis x-ray source, and detailed TALIS review of unconverted light, target alignment, and IP protection. B) IP
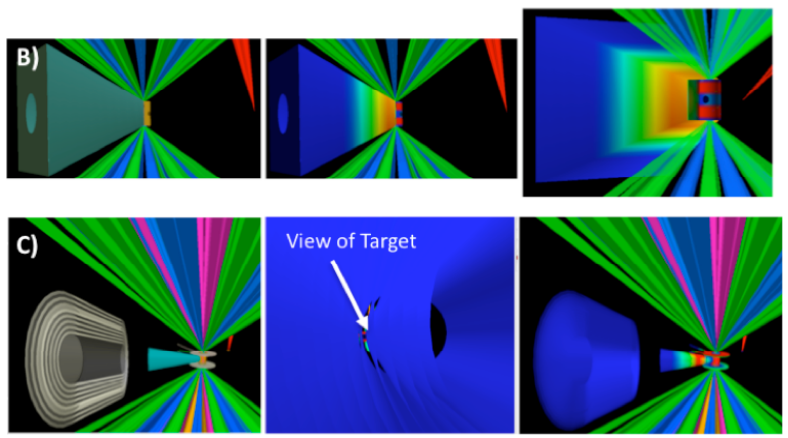

pyramid, using the NIF's Hohlraum geometry, including an off-axis x-ray source. Requires a detailed TALIS review of unconverted light, target alignment, and IP protection. C) Single IP shielded by conical Soller slits, using the NIF's Hohlraum geometry, including an offaxis $\mathrm{x}$-ray source, and avoiding all unconverted light. Requires a detailed TALIS review of diagnostic (Soller-slit) alignment.

The diamond diffraction targets will be manufactured by Diamond Materials $\mathrm{GmbH}$ in Germany. The target box (with associated debris calculations) used for holding image plates is being developed in concert with the materials IET. We will also attempt to recover the sample using schemes under consideration and development by the materials IET.

\section{Results Expected:}

We will monitor the phase-transition sequence of diamond on compression using $x$-ray diffraction to 30 Mbar. If as predicted, the BC8 phase is not seen on compression, we 
will perform a compress-release-hold experiment to look for BC8. We will also attempt a recovery experiment to look for super-hard metastable phases of carbon.

\section{Desired Platform:}

The platform will be similar to the HED materials platform as described on the NIF experimental platform webpage.

\section{Experimental Team:}

Professor Justin Wark, University of Oxford, justin.wark@physics.ox.ac.uk, UK citizen.

Dr. Jon Eggert, LLNL, eggert1@IInl.gov, US citizen.

Dr. Dennis Klug, National Research Council of Canada, Dennis.Klug@nrc.ca, US citizen.

Dr. Yansun Yao, National Research Council of Canada, Chinese citizen.

Professor Choong-Shik Yoo, Washington State University, csyoo@wsu.edu, US citizen.

Dr. Andrew Higginbotham, University of Oxford,

Andrew.Higginbotham@physics.ox.ac.uk, UK citizen.

Matthew Suggit, grad-student, University of Oxford, m.suggit1@physics.ox.ac.uk, UK citizen.

Gabriel Mogni, grad-student, University of Oxford, g.mogni1@physics.ox.ac.uk, Italian citizen.

Nigel Park, AWE Aldermaston, Nigel.park@awe.co.uk, UK UK citizen.

Professor Keisuke Shigemori, Osaka University, shige@ile.osaka-u.ac.jp, Japanese citizen.

Professor Katsuya Shimizu, Osaka University, shimizu@cqst.osaka-u.ac.jp, Japanese citizen.

Dr. Tom Boehly, University of Rochester, trb@lle.rochester.edu, US citizen.

Dr. Ryan Rygg, LLNL, rygg1@IInl.gov, US citizen.

Dr. James Hawreliak, LLNL, hawreliak1@IInl.gov, UK citizen.

Dr. Dan Kalantar, LLNL, kalantar@IInl.gov, US citizen.

Dr. Damien Hicks, LLNL, hicks13@IInl.gov, Australian citizen.

Dr. Peter Celliers, LLNL, celliers1@IInl.gov, Canadian citizen.

Dr. Hye-Sook Park, LLNL, Park1@IInl.gov, US citizen.

Dr. Gilbert Collins, LLNL, collins7@IInl.gov, US citizen.

Dr. Bruce Remington, LLNL, remington2@IInl.gov, US citizen.

In addition, this proposal is submitted in concert with all members of the Planetary Sciences University Use of NIF Consortium led by Raymond Jeanloz, UC Berkeley, jeanloz@berkeley.edu

\section{Required Capabilities and Resources:}

We believe we will need about 5 NIF shots per year over the 3-year period FY 2011-2013 to perform these experiments. 


\section{References:}

1 J. H. Eggert, D. G. Hicks, P. M. Celliers, D. K. Bradley, R. S. McWilliams, R. Jeanloz, J. E. Miller, T. R. Boehly, and G.W. Collins, "Melting temperature of diamond at ultrahigh pressure," Nature Physics 6, 40-43 (2010).

2 R. S. McWilliams, J. H. Eggert, D. G. Hicks, D. K. Bradley, P. M. Celliers, D. K. Spaulding, T. R. Boehly, G. W. Collins, and R. Jeanloz, "Strength effects in diamond under shock compression from 0.1 to 1 TPa," Phys. Rev. B 81, 014111 (2010).

3 D. K. Bradley, J. H. Eggert, R. F. Smith, S. T. Prisbrey, D. G. Hicks, D. G. Braun, J. Biener, A.V. Hamza, R. E. Rudd, and G.W. Collins, "Diamond at 800 GPa," Phys. Rev. Lett. 102, 075503 (2009).

4 D. G. Hicks, T. R. Boehly, P. M. Celliers, D. K. Bradley, J. H. Eggert, R. S. McWilliams, R. Jeanloz, and G. W. Collins, "High precision measurements of the diamond Hugoniot in and above the melt region," Physical Review B 78, 174102 (2008).

5 Stéphanie Brygoo, Emeric Henry, Paul Loubeyre, Jon Eggert, Michel Koenig, Bérénice Loupias, Alessandra Benuzzi-Mounaix, and Marc Rabec le Gloahec, "Laser-shock compression of diamond and evidence of a negative slope melting curve.," Nature Materials 6, 274-277 (2007).

6 D.K. Bradley, J.H. Eggert, D.G. Hicks, P.M. Celliers, S.J. Moon, R.C. Cauble, and G.W. Collins, "Shock Compressing Diamond to a Conducting Fluid," Phys. Rev. Lett. 93, 195506 (2004).

7 M. D. Knudson, M. P. Desjarlais, and D. H. Dolan, "Shock-Wave Exploration of the HighPressure Phases of Carbon," Science 322, 1822-1825 (2008).

8 H. Nagao, K.G. Nakamura, K. Kondo, N. Ozaki, K. Takamatsu, T. Ono, T. Shiota, D. Ichinose, K. A. Tanaka, K. Wakabayashi, K. Okada, M. Yoshida, M. Nakai, K. Nagai, K. Shigemori, T. Sakaiya, and K. Otani, "Hugoniot measurement of diamond under laser shock compression up to $2 \mathrm{TPa}$ " Phys. Plasmas 13, 052705 (2006).

9 W. J. Nellis, A. C. Mitchell, and A. K. McMahan, "Carbon at pressures in the range 0.1-1 TPa (10 Mbar)," J. Appl. Phys. 90, 696 (2001).

10 A.A. Correa, S.A. Bonev, and G. Galli, "Carbon under extreme conditions: Phase boundaries and electronic properties from first-principles theory " Proc. Nat. Acad. Sci. 103, 1204-1208 (2006).

11 S. Scandolo, G. L. Chiarotti, and E. Tosatti, "SC4: A metallic phase of carbon at terapascal pressures," Phys. Rev. B 53 (9), 5051-5054 (1996).

12 Jian Sun, Dennis D. Klug, and Roman Martoňák, "Structural transformations in carbon under extreme pressure: Beyond diamond," J. Chem. Phys. 130, 194512 (2009).

13 R.H. Wentorf, Jr. and J.S. Kasper, "Two new forms of silicon," Science 139, 338-339 (1963).

14 K. B. Fournier, J. H. Satcher, M. J. May, J. F. Poco, C. M. Sorce, J. D. Colvin, S. B. Hansen, S. A. MacLaren, S. J. Moon, J. F. Davis, F. Girard, B. Villette, M. Primout, D. Babonneau, C. A. Coverdale, and D. E. Beutler, "Absolute x-ray yields from laser-irradiated germaniumdoped low-density aerogels," Phys. Plasmas 16, 052703 (2009).

15 Dayne Fratanduono and et al., In Preparation (2010).

16 M. Mezouar, P. Faure, W. Crichton, N. Rambert, B. Sitaud, S. Bauchau, and G. Blattmann, "Multichannel collimator for structural investigation of liquids and amorphous materials at high pressures and temperatures," Rev. Sci. Instrum. 73, 3570-3574 (2002). 


\section{Summary of proposed experiment (Page 1 of 3)}

-Desired platform (If known): Materials

(Available platforms include: a) Capsule implosions; b) Hohlraum energetics;

c) Radiation transport; d) Shock timing; e) Streaked radiography; f) X-ray opacity; g)

$X$-ray sources. For further information on platforms see the NIF website: https://

lasers.IInl.gov/for_users/experimental_capabilities/index.php)

- Number of shots requested: Please fill out table below indicating number of "good data" shots requested each year. Do not add in additional shots to account for contingency, experimental problems, etc; NIF staff will consider this in planning evaluation

\begin{tabular}{|l|c|c|c|l|}
\hline $\begin{array}{l}\text { Summary Shot } \\
\text { Table }\end{array}$ & FY2010 & FY2011 & FY2012 & Comments \\
\hline Total shots & 0 & 5 & 5 & \\
\hline
\end{tabular}




\section{Summary of proposed experiment (Page 2 of 3)}

The National Ignition Facility

- Brief campaign description (include summary of preparatory shots (drive, diagnostic development, other) and actual data acquisition shots):

-2010-We will converge on a diagnostic that will make it through the TALIS review. We have 3 conceptual designs and have begun to evaluate the feasibility of each.

-2011-We will develop and impliment the chosen diagnostic and begin to take diffraction data. At first we will reproduce the data that we have achieved at the Omega and Omega EP facilities up to $10 \mathrm{Mbar}$. Afterwards we will attempt to drive and measure diffraction up to 25 Mbar

-2012-We will continue to explore the high pressure diffraction of new phases of diamond. If the BC8 phase is not observed on compression to $25 \mathrm{Mbar}$ we will develop a ramp and release design to look for the BC8 phase on release.

-We expect to be able to achieve these goals with $\mathbf{5}$ shots per fiscal year. 


\section{Summary of proposed experiment (Page 3 of 3)}

- Sketch of experimental configuration: Pls. provide a simple sketch of the experimental configuration below. Include orientation of target, laser and any backlighter beams, diagnostic sightlines, etc. If configuration is identical to an existing platform so indicate. For further information on existing platforms and chamber geometry see the NIF website:

https://lasers.IInl.gov/for_users/experimental_capabilities/index.php
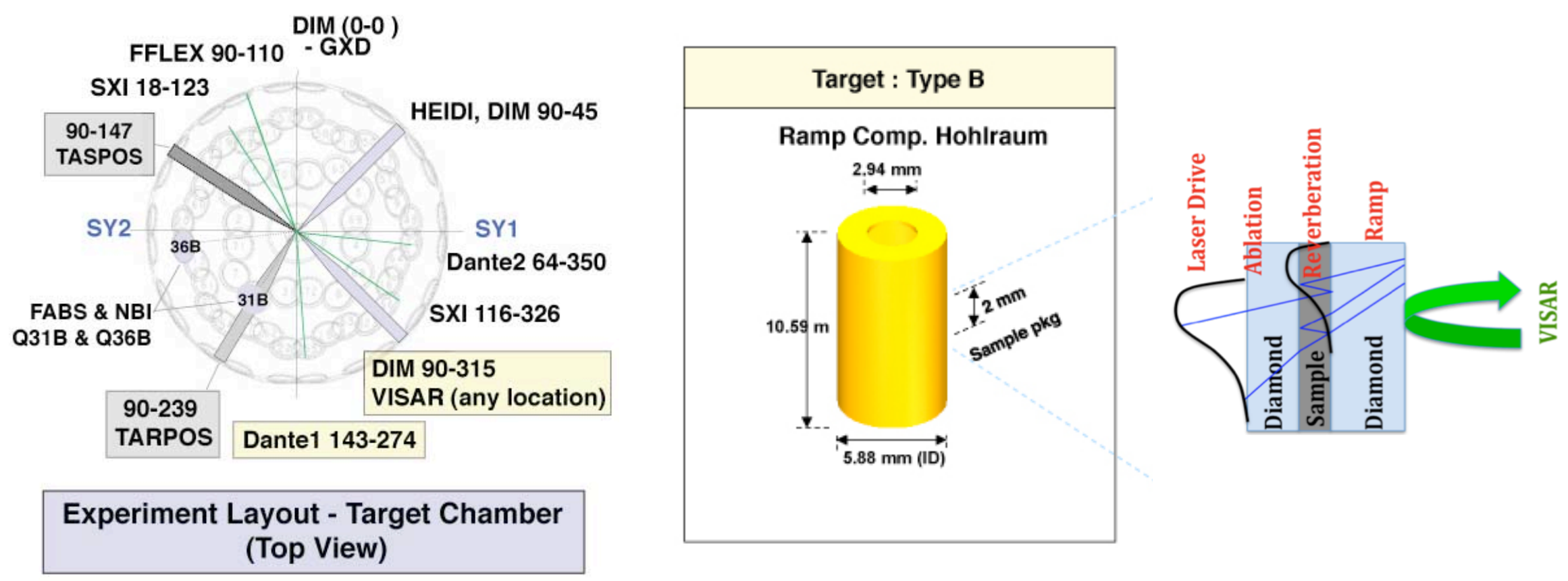

Experimental set-up: One for each unique illumination AND diag config, 


\section{Possible diagnostic configurations}
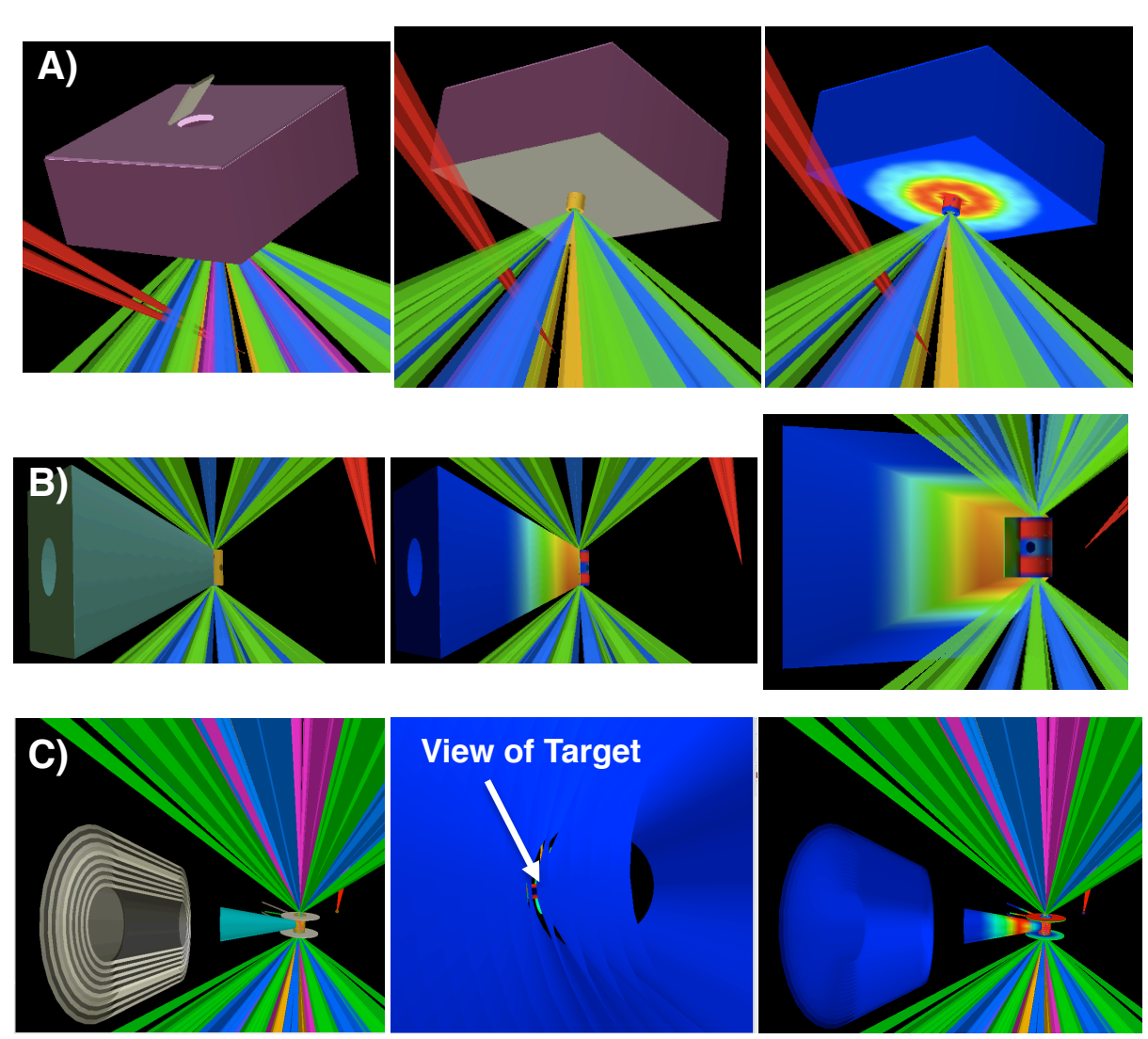

Three possible configurations for an x-ray diffraction diagnostic on the NIF. A) IP box with hemispherical symmetry. Requires a $90^{\circ}$ VISAR mirror, on-axis $x$-ray source, and detailed TALIS review of unconverted light, target alignment, and IP protection. B) IP pyramid, using the NIF's Hohlraum geometry, including an off-axis X-ray source. Requires a detailed TALIS review of unconverted light, target alignment, and IP protection. C) Single IP shielded by conical Soller slits, using the NIF's Hohlraum geometry, including an off-axis x-ray source, and avoiding all unconverted light. Requires a detailed TALIS review of diagnostic (Sollerslit) alignment. 


\section{Diagnostic requirements}

-Please refer to the diagnostic list on NIF user website:

https://lasers.IInl.gov/for_users/experimental_capabilities/diagnostics.php

- List below NIF diagnostics required for your experiment (along with a short summary description of required spatial, temporal, and spectral resolution) or describe what you wish to observe, and NIF staff will match to available diagnostics.

-VISAR/SOP

-DANTE

-SXI

-FABs/NBI

-FFLEX

- Also indicate below if any additional, user provided diagnostics are required.

Provide a short summary of the user provided diagnostic below, including a list of all materials to be introduced into the target chamber.

-NIF XRD-Xray Diffraction Diagnostic to be developed. This diagnostic will introduce Tantalum, Stainless Steel, and Image Plates into the target chamber. We will develop a diagnostic consistent with NIF requirements 


\section{Laser requirements (1 of 2)}

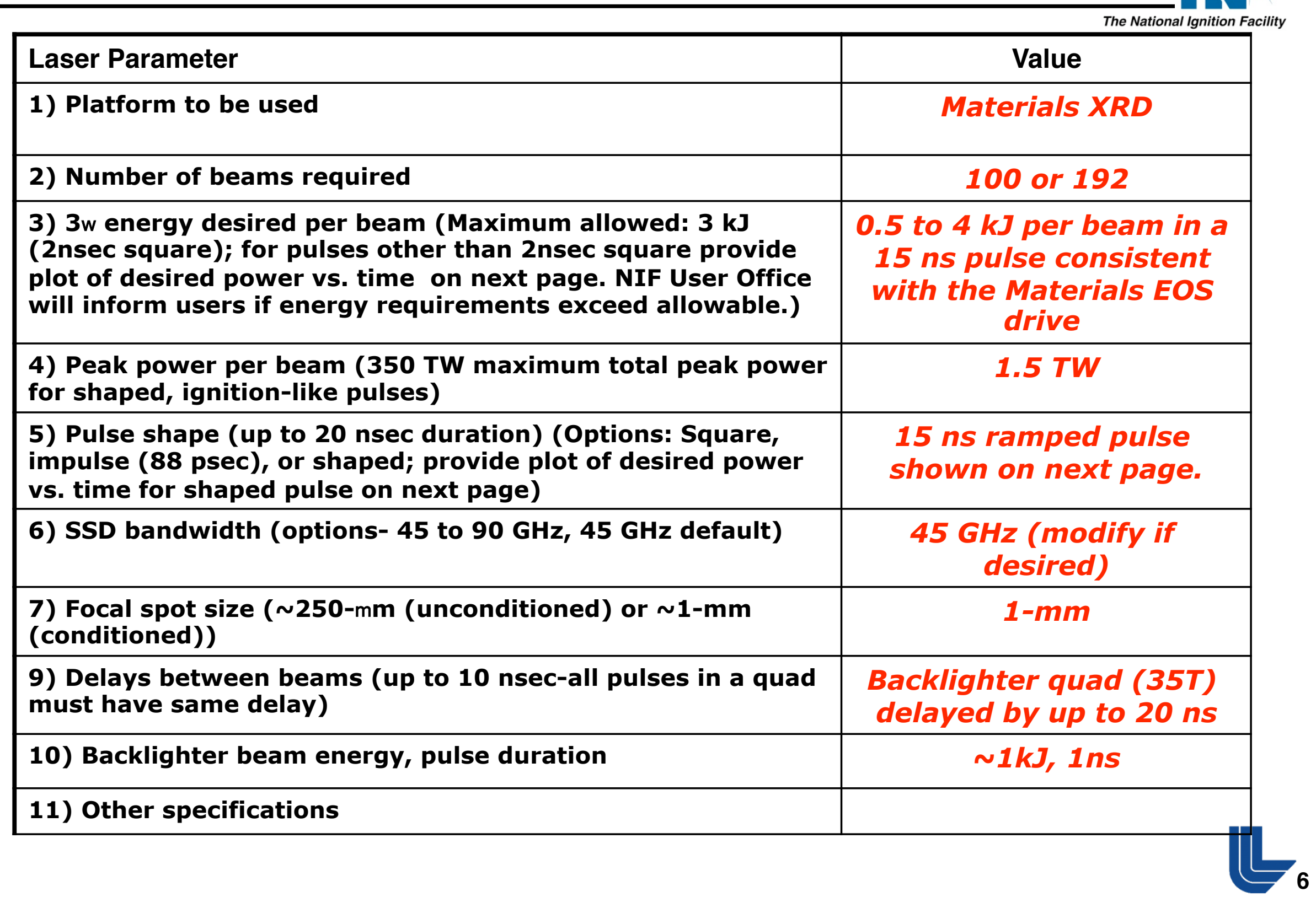




\section{Laser requirements (2 of 2)}

For shaped pulses, sketch desired power vs. time below:

This is the maximum pulse per beam required. Shots will be range from about 10 to $100 \%$ of this.

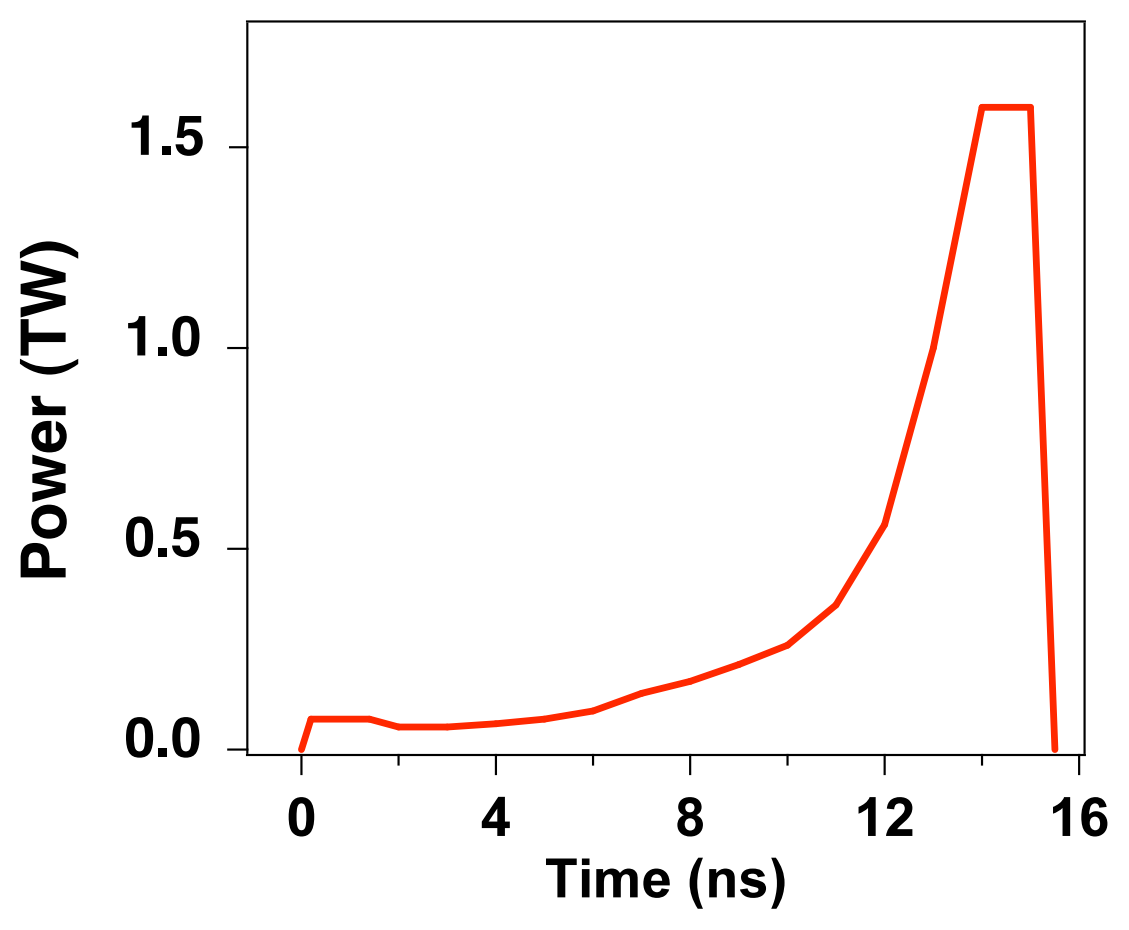




\section{Target requirements (1 page per target type)}

- List target types required (example: drive measurement; diagnostic test; data acquisition target)

We will use both diamond and LiF final windows for our targets. The package is $3 \mathrm{~mm}$ diameter, and the tantalum pinhole is $5 \mathrm{~mm}$ diameter.

- For each target type provide a sketch of the target below. Include dimensions and a list of all materials to be used. Also indicate any critical tolerances required, and indicate components (if any) to be provided by the Principal Investigator.
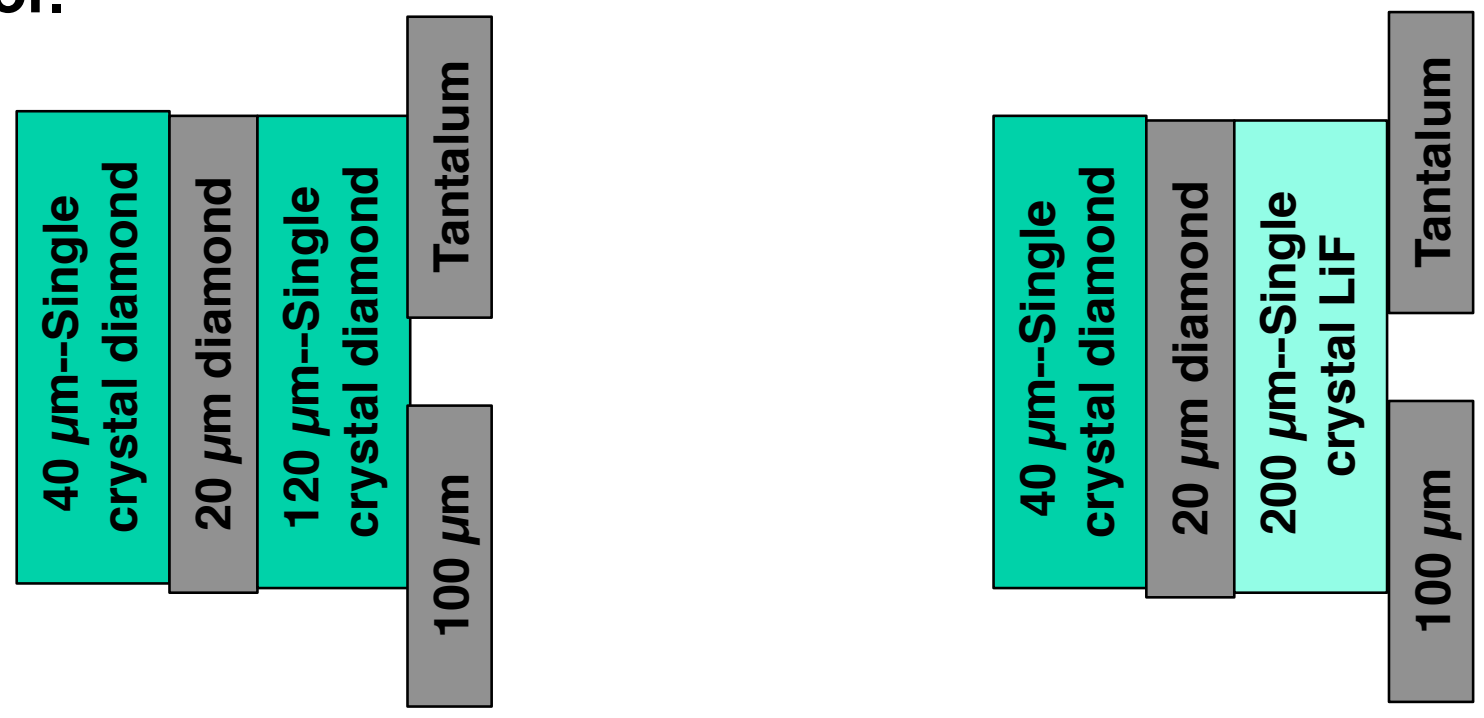